\title{
An Evaluation Model for The Impact of Climate Change on Regional Instability
}

\author{
Jia-Yi Lie ${ }^{1}$, Hong-Yu Duan ${ }^{1, a}$ and Dong Chen ${ }^{1}$ \\ ${ }^{1}$ Jinan University, China
}

\begin{abstract}
The complication that climate change brings about has been an important issue in recent decades. In this paper, we attempt to find the inner connection between climate change and regional instability, and finally build a novel evaluation model about it. By innovatively utilizing Analytic Hierarchy Process and Entropy Weight Method combined, our model can produce a reliable climate change impact assessment. Empirical results on states of different stability provide strong evidence that our model possesses high feasibility and accuracy in practical use.
\end{abstract}

\section{Introduction}

In recent years, the tremendous effects of climate changes have been people's major concern. Not only can they alter the way we live, but they are also likely to ruin our social structures, turning destabilized governments into fragile states.

A fragile state means the state government is not able to provide necessities to its people. Thus, people who live in such state tend to suffer more in climate shocks or violent conflicts that result from natural disasters. According to The Intergovernmental Panel on Climate Change, climate change will have a significant influence on the fragility of a country. So, the possible linkage between climate change and regional instability has been a new subject in meteorology and sociology. In this paper, we propose a model to determine a country's fragility.

To build the relationship between climate change and state instability, we firstly develop a model that determines a country's fragility and measure the influence of climate change. Here we assume that in short term there won't be any huge changes in both climatic and political conditions. Then we design three climatic factors as well as twelve indicators to appraise a state's fragility. We think that climate influence the state instability indirectly via the twelve state fragility indicators. For example, the flood of drought which are caused by extreme precipitation will affect the crops and buildings, thus does harm to economic development. Combining all the indicators in Fragile States Index, we can construct a three-layer indicator system. Next, we apply AHP-EWM fusion method to obtain the weight vectors of indicators in each layer. Meanwhile, two tipping points can be obtained through K-means clustering method. An evaluation system, which can identify when a state is fragile, vulnerable, or stable, is eventually built.

In order to demonstrate the effectiveness of our model, we apply it to Afghanistan and Kenya respectively.

\footnotetext{
a Corresponding author: 932605498@qq.com
}

Afghanistan is among the 10 most fragile states. By comparing its fragility index tendency during the past 12 years with its fsi in The Fund for Peace, we can safely conclude that climate does affect the fragility in Afghanistan. As for Kenya, we employ BP Neural Network for autoregression on the indicators and find that Kenya may reach its tipping point in 2020 when the climatic factors will generate a serious influence on state fragility.

At last, we briefly analyse how human interventions are likely to influence the Fragility Index and make some modifications which enable our model to perform better in different conditions.

\section{Model Theory}

\subsection{Climatic Factors}

It is universally acknowledged that surface air temperature (hereinafter referred as temperature) and precipitation are the two most essential factors in climate study. Therefore, we will analyse the two factors respectively.

\subsubsection{Precipitation}

The annual rainfall is an important measurement of regional precipitation. Too much rainfall may lead to disasters such as flood and landslide; whereas too little can lead to water shortage, which will surely do harm to crops. By referring to relevant information, we get to know that $1000 \mathrm{~mm}$ is the ideal annual precipitation for urban development and resident life both.

So, we define the Precipitation Index as:

$$
P I^{\prime}=|P-1000|
$$

Where $P$ represents the annual precipitation in a certain region. 
After rescaling, we can get the normalized Precipitation Index:

$$
P I=\frac{P I^{\prime}-P I_{\text {min }}^{\prime}}{P I_{\text {max }}^{\prime}-P I_{\text {min }}^{\prime}}
$$

\subsubsection{Temperature}

We usually use the annual mean temperature to measure the temperature. It's apparent that the temperature, neither too high or too low, will be bad for the city development. According to relevant material, $80^{\circ} \mathrm{F}$ is the most suitable temperature for urban development and resident life. Thus, we define the Temperature Index as:

$$
T I^{\prime}=|T-80|
$$

Where $T$ denotes the annual mean temperature in a certain region.

By implementing the rescaling method, we will get the normalized Temperature Index:

$$
T I=\frac{T I^{\prime}-T I_{\text {min }}^{\prime}}{T I_{\max }^{\prime}-T I_{\text {min }}^{\prime}}
$$

\subsubsection{Extreme Climate}

Apart from normal climate, extreme climate also has a huge effect on the city. Theoretically, extreme climate must fulfil either of conditions below [1]:

a) Temperature $>86^{\circ} \mathrm{F}$ or temperature $<32^{\circ} \mathrm{F}$

b) Precipitation $>2 \mathrm{~mm}$ or precipitation $=0$

It's obvious that the less extreme climate there is, the better the city develops. Therefore, we define the Extreme Climate Index as follows:

$$
E I^{\prime}=E
$$

After rescaling, the normalized Extreme Climate Index is:

$$
E I=\frac{E I^{\prime}-E I_{\text {min }}^{\prime}}{E I_{\text {max }}^{\prime}-E I_{\text {min }}^{\prime}}
$$

To better discuss the follow-up problems, here we assume that smaller indexes indicates better environment for states because when the three indexes above rises, the states can be more fragile.

\subsection{State Fragility Indicators}

A fragile state is one where the government is not able to, or chooses not to, provide the essentials to its people. Generally, the state fragility is decided by four aspects, namely cohesion, economy, politics, and society [2].

\subsubsection{Cohesion (CON)}

C1 Security Apparatus: Home land security will influence the fragility of a state

C2 Factionalized Elites: The influence of a few elite can be enormous

C3 Group Grievance: Some groups possess great control over the development situations

\subsubsection{Economy(ECO)}

E1 Economy: Economy is of vital significance in the development of a country

E2 Economical Inequality: Unequal economic development will weaken social and governmental structures

E3 Human Flight and Brain Drain: Human flight and brain drain may further aggravate state fragility

\subsubsection{Politics (POL)}

P1 State Legitimacy: A country with complete legislation would have higher stability

P2 Public Services: Public services will affect the stability of a country

P3 Human Rights: Human rights guarantee the national stability

\subsubsection{Society(SOC)}

S1: Demographic Pressures: Problems such as overpopulation and an aging population will undermine the stability of the country

S2: Refugees and IDPs: Increase refugees exacerbates the country's vulnerability

X1: External Intervention: External intervention puts pressure on the government

\subsection{Three-Layer Indicator System}

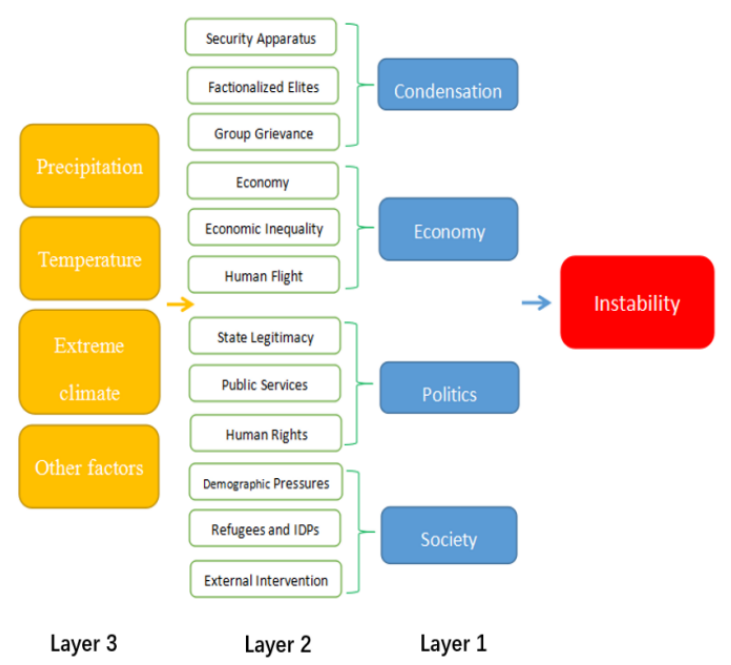

Figure 1. Hierarchical Diagram

Figurel depicts the linkage between climate change and state fragility. Here other factors in the figure refer to factors other than climate. All the factors and indicators are classified into three layers. Since we believe that other factors are mainly related to comprehensive national strength, especially GDP, we will roughly replace it with the normalized GDP reciprocal.

In order to study the relationship among climatic factors, other factors, state fragility indicators and state instability, in the next part we will combine the two commonly used methods of weight analysis - AHP and entropy weight method [3]. 


\subsection{AHP-EWM Fusion Method}

We acquire judgment matrix in AHP from experts by empirical rating process, which makes it rather difficult to eliminate the subjective factors. By contrast, entropy weight method calculates the weight based on objective data, but it reflects the relative intensity of competition between indicators rather than the actual importance.

AHP-EWN fusion method innovatively combine the two methods while calculating the weight. The detailed steps are as follows.

Suppose that there are $\mathrm{x}$ indicators in Layer $1, m$ indicators in Layer 2 and $\mathrm{n}$ indicators in Layer 3. each indicator in Layer 2 contains $\mathrm{n} 1, \mathrm{n} 2, \ldots, \mathrm{nm}$ sub-indicators separately and $\mathrm{n} 1+\mathrm{n} 2+\ldots+\mathrm{nm}=\mathrm{n}$.

\section{Step 1}

Establish the judgement matrix through AHP, and we'll get the weight vector of indicators in Layer $1: A=$ $\left\{a_{1}, a_{2}, \ldots, a_{\mathrm{n}}\right\}$; the weight vector of indicators in Layer 2 : $B=\left\{b_{1}, b_{2}, \ldots, b_{\mathrm{n}}\right\}$; the weight vector of indicators in Layer $3: C=\left\{c_{1}, c_{2}, \ldots, c_{\mathrm{n}}\right\}$.

\section{Step 2}

Apply the entropy weight method and we can obtain the weight of each indicator: $U=\left\{u_{1}, u_{2}, \ldots, u_{\mathrm{n}}\right\}$.

\section{Step 3}

Synthesize the weight vectors of indicators in Layer 3 obtained from AHP and EWM, then we can get the integrated weight vector: $T=\left\{t_{1}, t_{2}, \ldots, t_{\mathrm{n}}\right\}$, where

$$
t_{j}=\frac{u_{j} c_{j}}{\sum_{j=1}^{n} u_{j} c_{j}}, j=1,2, \cdots, n
$$

\section{Step 4}

According to the correspondence with indicators of Layer 2, we refine the integrated weight vector of indicators in Layer 3 and get

$$
T=\left\{t_{11}, t_{12}, \ldots, t_{1 \mathrm{n}}, t_{21}, t_{22}, \ldots, t_{2 \mathrm{n}}, \ldots, t_{\mathrm{m} 1}, t_{\mathrm{m} 2}, \ldots, t_{\mathrm{mn}}\right\}
$$

After applying rescaling method to the weight vectors of each Layer 3 indicator within Layer 2, we can have: $D=\left\{d_{11}, d_{12}, \ldots, d_{1 \mathrm{n}}, d_{21}, d_{22}, \ldots, d_{2 \mathrm{n}}, \ldots, d_{\mathrm{m} 1}, d_{\mathrm{m} 2}, \ldots, d_{\mathrm{mn}}\right\}$

Where

$$
d_{i j}=\frac{t_{i j}}{\sum_{j=1}^{k} t_{i j}}, i=1,2, \cdots, m ; k=n_{1}, n_{2}, \cdots, n_{m} .
$$

\section{Step 5}

Multiply the corresponding weight in vector $B$ and vector $D$ and we will get

$E=\left\{e_{11}, e_{12}, \ldots, e_{1 \mathrm{n}}, e_{21}, e_{22}, \ldots, e_{2 \mathrm{n}}, \ldots, e_{\mathrm{m} 1}, e_{\mathrm{m} 2}, \ldots, e_{\mathrm{mn}}\right\}$

where $\mathrm{e}_{\mathrm{ij}}=b_{\mathrm{ij}} \times \mathrm{d}_{\mathrm{ij}}$.

\section{Step 6}

Replace weight $E$ with $F=\left\{f_{1}, f_{2}, \ldots, f_{\mathrm{n}}\right\}$ for simplification. After applying rescaling method to $F$,we can get the ultimate weight $\mathrm{W}=\left\{w_{1}, w_{2}, \ldots, w_{\mathrm{n}}\right\}$, in which

$$
w_{j}=\frac{f_{j}}{\sum_{j=1}^{n} f_{j}}, j=1,2, \cdots, n
$$

In this way, we can build the relationship between state instability and climate change in nearly every country. However, it is noticeable that the equation varies from country to country. That is:

$$
F I^{\prime}{ }_{i}=w_{1 \mathrm{i}} P I_{\mathrm{i}}+w_{2 \mathrm{i}} T I_{\mathrm{i}}+w_{3 \mathrm{i}} E I_{\mathrm{i}}+w_{4 \mathrm{i}} O I_{\mathrm{i}}
$$

After normalization we have:

$$
F I=\frac{F I^{\prime}-F I^{\prime}{ }_{\text {min }}}{F I_{\text {max }}^{\prime}-F I_{\text {min }}^{\prime}}
$$

Where $w=\left[w_{1 \mathrm{i}}, w_{2 \mathrm{i}}, w_{3 \mathrm{i}}, w_{4 \mathrm{i}}\right]$ represents the weight vector of the $i^{\text {th }}$ country. Here $i$ means the $i^{\text {th }}$ country.

\subsection{Instability Assessment}

We plug in precipitation index, temperature index, extreme climate index and other-factor index from 20 countries to get their weights respectively. Then we take the mean value of the weights from the 20 countries as our standard weights. We represent the different weights in table 1 and table 2(Because there are too many indicators in Layer 2, we omit them in the table below)

Table 1. Weight of Indicators in Layer 1 and Layer 3

\begin{tabular}{l|l}
\hline Factor & Weight \\
$C O N$ & 0.1556 \\
$E C O$ & 0.4301 \\
$P O L$ & 0.2341 \\
SOC & 0.1802 \\
\hline
\end{tabular}

\begin{tabular}{l|l}
\hline Factor & Weight \\
$P I$ & 0.0352 \\
$T I$ & 0.0142 \\
$E I$ & 0.4034 \\
$O I$ & 0.5472 \\
\hline
\end{tabular}

The AHP has already passed the consistency test. So, we correct (1) and get:

$$
F I^{\prime}=w_{1} P I+w_{2} T I+w_{3} E I+w_{4} O I
$$

Where the weigh vector is $\mathrm{w}=(0.0352,0.0142$, $0.4034,0.5472)$. The rescaling method remains unchanged, which is suitable for all countries.
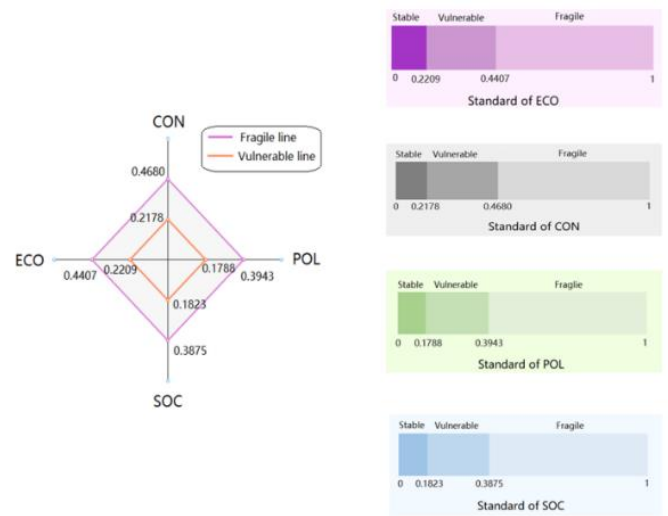

Figure 2. The Tipping Point of Indicators in Layer 1

Then we apply K-means clustering method by using $\mathrm{K}$-means function in MATLAB, where $K=3$. We divide the data into three categories according to the four 
indicators in Layer 1 and get three class centres. Define their midpoints as tipping points and put them into equation (2), and we'll get the tipping point of FI, namely 0.2036 and 0.4245 .

Hence, we get two methods to determines a country's fragility:

A) Use the tipping points of indicators in Layer 1 as criterion. The detailed measuring standard is shown in figure 2 .

B) Use the tipping point of $F I$ as a criterion. If FI falls on $[0,0.2036]$, the country is stable. If on [ 0.2036 , $0.4245]$, the country is in a vulnerable state. FI on [ $0.4245,1]$ denotes a fragile state. The measuring standard is presented in

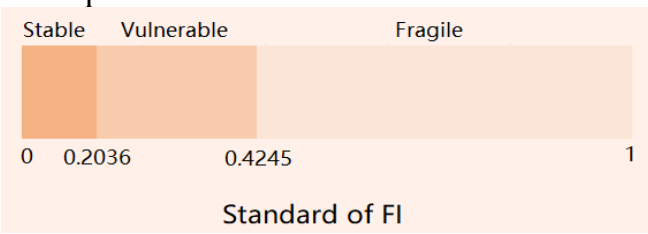

Figure 3. Classification of State Fragility

\subsection{Data Collecting and Processing}

All the statistics that we use in 4.4 as well as the following sections are originated from Worldbank [4].

Data availability is a fundamental issue in model implementing. Valid assessment can never be achieved with unreliable or unrealistic data, no matter what measures have been taken. Therefore, it is of paramount importance to ensure the continuity and authenticity of the data. Due to information incompetence and data loss on the internet, we use the following methods to supplement these data:

If the indicator value is stable, we can use the previous data for supplement

If we can get the data on both sides of the missing one, we will fill in the average.

If two data are similar, the missing data can be replaced by the corresponding value in the other group.

In other cases, we will employ polynomial interpolation for data fitting.

\section{Model Validation}

To validate that our model, here we choose two countries, namely Afghanistan and Kenya, and apply our model to them respectively.

\subsection{Analysis on Afghanistan}

Among the top 10t most fragile countries [5], we select Afghanistan for implement. From Worldbank we can obtain the climate data of Afghanistan in the past 12 years. Then we put the data into the model that we build in section 3.3. Below are the results that we have get in MATLAB:

As is shown in the picture, the FI of Afghanistan is always in [ 0.41, 0.47] , which means that Afghanistan is in a fragile state. Moreover, we can get the total fsi of
Afghanistan in the last 12 years from The Fund for Peace[4]. Its trend is presented in the curve below.
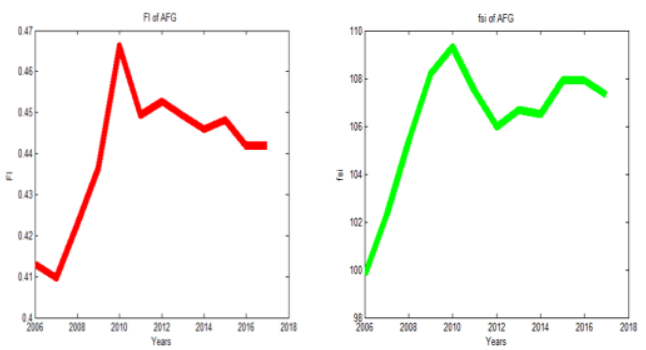

Figure 4. FI Tendency Chart of Afghanistan and Trend of Total fsi in Afghanistan

By comparison, it's easy to find that their trend is highly consistent, which proves that our model is effective and reliable.

According to our model, when indexes of precipitation, temperature or extreme climate varies, there is a correspondence between their effect on fragility and their coefficients in equation (2). For example, when the precipitation condition gets better and $P I$ decreases 1 unit, the fragility indicator will reduce 0.0352 unit.

Next, we try to eliminate the effects of climate. Supposing that the three factors remain unchanged, we replace them with the corresponding value in 2006.Then we plot the tendency of FI in figure 5 .

By analysing the fluctuation of $F I$, we find that the growth of $F I$ is slower when the climate is set as unchanged. In certain period, it is even on the decrease. So, we can see that the growth of its fragility has been weakened

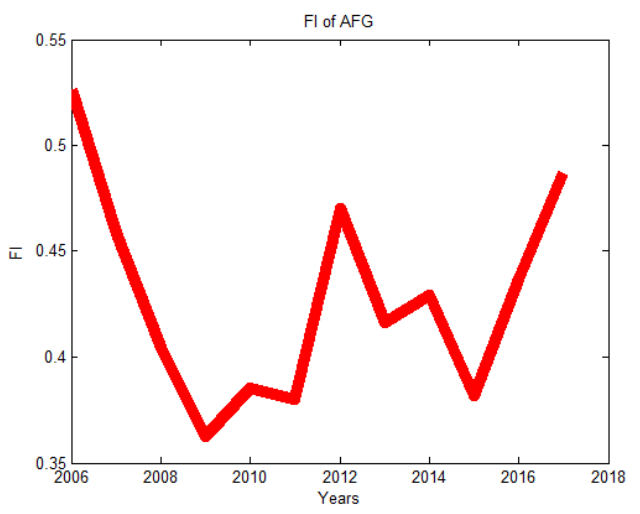

Figure 5. The Tendency of FI in Afghanistan With the Climate Unchanged

\subsection{Analysis on Kenya}

Located in the eastern Africa, the precipitation in Kenya varies from season to season. What's worse, the precipitation in Kenya is getting less and less because of global warming. We can obtain from Worldbank the climate data in Kenya during the latest 12 years. Plug the data in our model, and the FI of Kenya in 2017 is $\mathrm{FI}_{2017}=$ 0.4076 . Additionally, we present the results of indicators in the figure below. So, Kenya is a vulnerable country. 

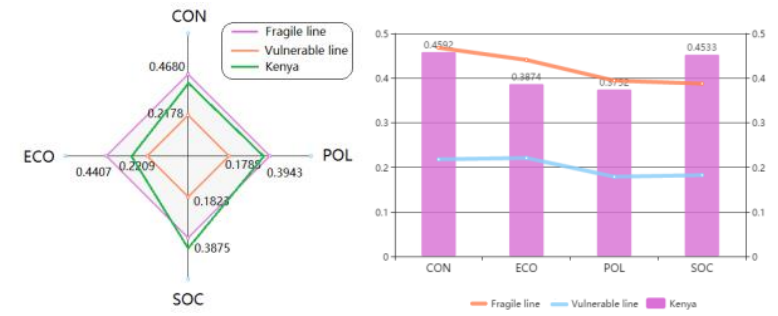

Figure 6. Results of Indicators in Layer 1 in Kenya in 2017

Plot the trend of three climatic factors of Kenya in MATLAB from 2006 to 2018:

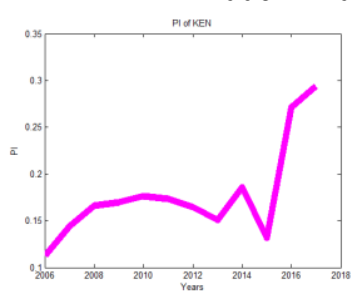

(a)

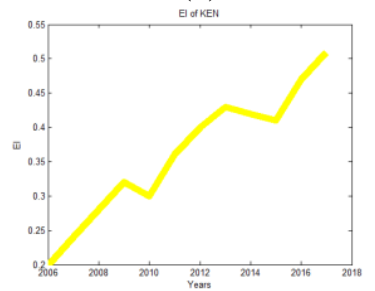

(c)

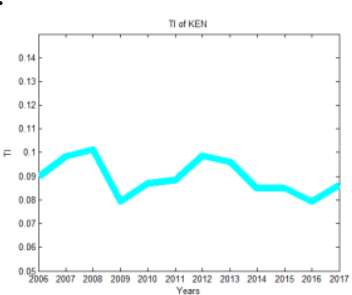

(b)
Figure 7. The Tendency Chart of Climatic Factors of Kenya

Where (a) shows the tendency of precipitation index, (b) for temperature index and (c) for Extreme Climate Index.

As the figure depicts, extreme precipitation occurs frequently in Kenya during the period. According to reports online [6-7], we find that in recent years the rainfall in Kenya fluctuate dramatically. Drought and flood threaten the lives of Kenyans in turn and slow down their economic and social development, resulting in the long-term instability in the country.

Because the temperature in Kenya remains steadily between $64{ }^{\circ} \mathrm{F}$ and $86^{\circ} \mathrm{F}$, which means it is not in an extreme condition, we mainly focus on the influence that precipitation has on the instability of Kenya.

\subsubsection{How to Find the Tipping Point}

In this section we meant to find the tipping point of days when extreme weather happens to distinguish the difference in influence, however failed. Thus, we define the mean value of extreme climate days in the ten most fragile countries, namely 55 days, as a tipping point that has a major impact on the country.

The majority of extreme climate is related to extreme rainfall intensity. So, we use BP neural network for the autoregression of extreme climate days in Kenya.

\subsubsection{A Brief Introduction of BP Neural Network}

Neural networks have the following characteristics.

a) Self-study ability and adaptability

b) Computational parallelism

c) Nonlinearity

d) Distributed storage

e) Robustness and fault tolerance

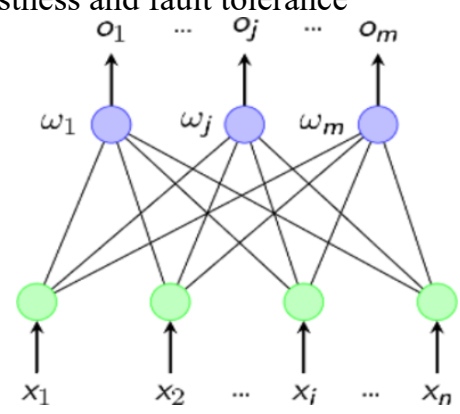

Figure 8. Schematic Diagram of Neural Network Structure

As the diagram shows, BP Neural Network is composed of the input layer $\left[x_{1}, x_{2}, \ldots, x_{\mathrm{n}}\right]$, the hidden layer $\left[\omega_{1}, \omega_{2}, \ldots, \omega_{\mathrm{n}}\right]$ and the output layer $\left[\sigma_{1}, \sigma_{2}, \ldots, \sigma_{\mathrm{n}}\right]$. The line across different layers represents the corresponding weights. For each data processing, we will apply the confirmed function to map and realize data fitting through the backpropagation mode of error signal. In this model, we select the number of hidden layer based on the formula below:

$$
m=\sqrt{n+l}+\alpha
$$

Where $n$ is the dimension of the input layer, $n$ is the dimension of the input layer, and $\alpha$ is the a constant between $[0,10]$. Here we will use training function "trainlm" as well as the transfer function "purelin" and "tansig".

\subsubsection{Regression by BP Neural Network}

Use MATLAB for data regression, and we'll get the results as figure 9 represented.

In figure 9, the blue part results from regression, while the red part comes from our predicted data.

It's not hard to find from figure 9 that with this the trend extreme precipitation will does great harm to Kenya in 2020. Therefore, the Kenyan government must immediately take measures to combat natural disasters.

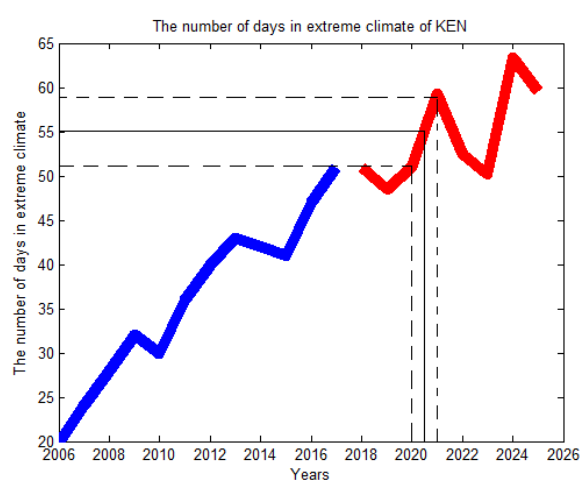

Figure 9. Autoregression Results of Extreme Climate Days in Kenya with BP Neural Network 
We then apply the BP Neural Network to get the data needed for the model. Solve it and we can get that FI2020 $=0.4332$. In addition, we plot the indicators in Layer 1 of Kenya in figure 10 and find that Kenya is in a fragile state, which demonstrates that our model is credible.
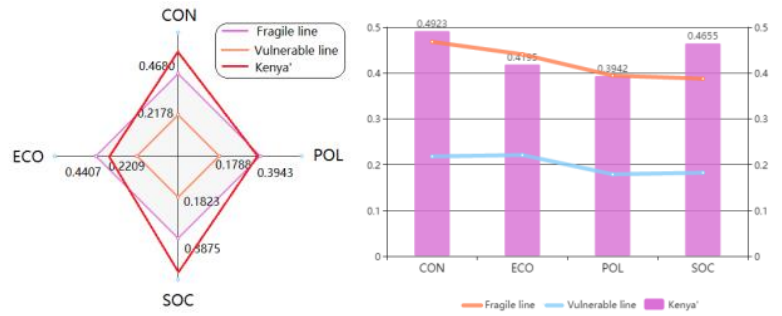

Figure 10. Results of Indicators in Layer 1 in Kenya in 2020

\subsubsection{Error Analysis}

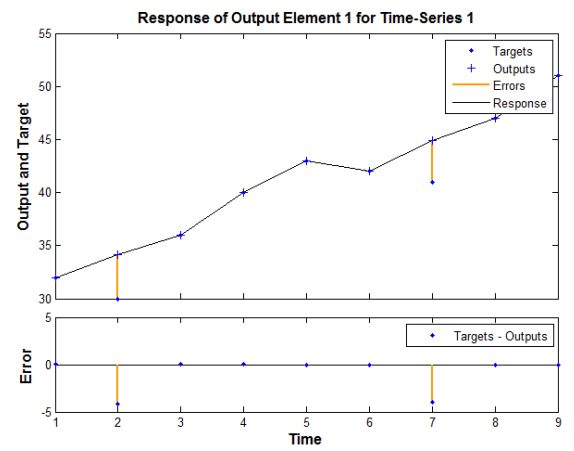

Figure 11. The Error Graph in the Regression

In this part we analyse the error during the regression process and draw the error graph above. We can see from the graph that the error during the regression is in $[0,5]$, which is in an acceptable range.

\section{The Effect of Human Interventions on State Fragility}

Since it's impossible to reduce the harm of extreme temperature change by massive human interventions, we only analyse drought and flood which are caused by extreme precipitation in this part.

\subsection{Measures Taken for Drought}

There are mainly two kinds of measures that can be adopted to solve the drought problem.

A) (Water) Resource Allocation. In some countries, drought frequently occurs in some regions but there are sufficient water resources in other regions. Then water in areas with adequate water resources can be directly diverted to areas where water is scarce. For example, China's south-north water diversion, Israel's south-north water diversion etc.

B) (Water) Resource Conservation/Generate. Some countries respond to drought by protecting or even generating resources. The resources are not only water, but also crops and other resources affected by drought.
For example, Egypt's legislation on water abuse, South Africa's groundwater desalination etc.

\subsection{Measures Taken for Flood}

The ultimate purpose of flood relief methods is to resist the hazard of flood. Sandbags, dams, and flood baffles are widely used in America, Japan, India, and Germany. Besides, human inventions such as expanding flood insurance coverage are also adopted in many countries. All these methods can decrease the influence of extreme precipitation, thus decrease the state fragility.

\subsection{The Effect of Human Intervention}

Firstly, we display the formula for FI above:

$$
F I^{\prime}=w_{1} P I+w_{2} T I+w_{3} E I+w_{4} O I
$$

We know that flood is caused by extreme precipitation. That is to say, decrease of extreme precipitation can promote flood mitigation and reduce its influence on the country. Thus, we reduce the value of $w_{3}$. Furthermore, measures such as expanding flood insurance coverage can directly reduce the impact of the flood disaster that caused by extreme precipitation. Then the value of $w_{3}$ will drop likewise. So, it's not difficult for us to draw the conclusion that human intervention can reduce $F I$, that is reducing the fragility of the country.

\section{Future work}

Due to the changes in climate zone, we need to make several modifications when applying our model to smaller or larger "states" (such as cities and continents).

\subsection{Modification for Smaller States}

As for smaller states (such as cities), the climate changes more frequently, which requires more detailed management. So, we modify our model in the following ways.

Firstly, we change the time unit of each indicator from "year" to "month". On the one hand, more statistics and information make it possible for our model to produce a more accurate result, thus providing more reliable instructions for the governors. On the other hand, short-term data is also better suited to small states which are far more demanding for accuracy.

Secondly, we collect the indicators of the small state itself along with those of all its neighbouring countries. Then we can take their weighted average as a final indicator. Because the small state and its neighbouring counties are in most cases located in the same climate zone, indicators of the neighbouring countries have a great reference value for the small country. The formula for the indicators is as follows.

$$
F I=0.7 \cdot F I_{i}+0.3 \cdot \frac{1}{n} \cdot \sum_{j \neq i} F I_{j}
$$


Where $\mathrm{n}$ represents the number of all the neighbouring countries, $i$ is the small country itself. We set the coefficients at 0.7 and 0.3 to ensure that the indicators of the small countries have the major influence on itself, whereas the influence of those in the neighbouring countries are relative small.

\subsection{Modification for Larger State}

Since there usually exist more than one climate zone and country in larger states, we can modify the model in the following way.

Set a significance index for each country in the large state and we'll get:

$$
S I=a S+b D+c E+d P
$$

Where S, D, E, P respectively means the normalized index of national land area, distance between state centre and country centre, economic strength and total population. As for the economic strength in the equation, we can roughly replace it with GDP per capita or solve it with fuzzy evaluation method [8]. The corresponding coefficient of every index can be obtained by AHP. Next, we assign a reasonable weight to each country using the following equation:

$$
w_{i}=\frac{S I_{i}}{\sum S I}
$$

Finally, combine the indexes of the countries and their corresponding weights, and we can get the indicators for large state by weighted average.

\section{Conclusion}

This article proposes a novel AHP-EWM fusion evaluation model, which focuses on the impact of climate change on regional instability. The proposed model is strictly based on meteorological facts and can achieve accurate assessment result. What's more, empirical studies show that our model can help people make predictions on regional instability. As an extension to the work, we make some brief modifications to ensure that our model is universally applicable to different states. In future, we will study more meteorology and take more factors into account in order to improve our model.

\section{References}

1. JiYun Xu, Ying Shi, XueJie Gao, GIORGI Filippo, Chinese Science Bulletin, 58 1443-1452 (2013)

2. Fragile https://en.wikipedia.org/wiki/Fragile_state/

3. YanLing Chen., Advances in Social Sciences, 6 728-734(2017)

4. world bank data, https://data.worldbank.org/, http://sdwebx.worldbank.org/climateportal/

5. The Fund for Peace, http://fundforpeace.org/

6. The drought in Kenya affects the lives of millions of people, http://www.xinhuanet.com/world/2017-02/17/c_129 482974.htm/

7. The floods in Kenya have caused the 100 thousand families to be affected, http://news.163.com/12/0517/06/81MG4S2V00014 AED.html/

8. LingYing Zhang, Systems Engi-neering Theory \& Practice, 7 98-101(1998) 